\title{
Comparison of Effects of Hemoglobin Levels Upon Tumor Response among Cervical Carcinoma Patients Undergoing Accelerated Hyperfractionated Radiotherapy versus Cisplatin Chemoradiotherapy
}

\author{
Priyanka Thakur, Rajeev Kumar Seam, Manoj Kumar Gupta, Madhup \\ Rastogi, Manish Gupta, Tapesh Bhattacharyya, Mukesh Sharma, Swaroop \\ Revannasiddaiah*
}

\begin{abstract}
Purpose: Blood hemoglobin levels are known to influence response to radiotherapy. This retrospective analysis compared the effect of hemoglobin levels upon response to radiation among patients treated with radiation alone (by accelerated hyperfractionated radiotherapy) versus those treated with concurrent cisplatin chemoradiotherapy. Materials and Methods: Among patients treated for locally advanced carcinoma of the cervix (LACC) during 2009-10, a total of 60 fulfilled the eligibility criteria. In this time frame, external beam radiotherapy was delivered with either concurrent chemoradiotherapy (CRT, n=31) (45Gy over 25 fractions, with weekly cisplatin at $40 \mathrm{mg} / \mathrm{m} 2)$, or with accelerated hyperfractionated radiotherapy (AHRT, n=29) (20Gy over 10 daily fractions over the first two weeks, followed by 30Gy over 20 fractions over the next two weeks, with two fractions of $1.5 \mathrm{~Gy}$ per day, without the use of chemotherapy). Mean weekly hemoglobin (MWH) levels of all patients were calculated as the arithmetic means of weekly recorded blood hemoglobin levels. As per MWH, patients in both of the AHRT or the CRT groups were classified into two subgroups- those with MWH between 10-10.9g/dL, or with MWH>11g/dL. Complete response (CR) to external beam RT phase (prior to brachytherapy) was declared after clinical examinations and computed tomography. The CR rate was noted for both MWH sub-groups within each of the AHRT and CRT groups. Results: Within the AHRT group, patients with $M W H>11 \mathrm{~g} / \mathrm{dL}$ had a much better $\mathrm{CR}$ rate in comparison to those with $\mathrm{MWH}: 10-10.9 \mathrm{~g} / \mathrm{dL}(\mathbf{8 0} \% \mathrm{vs.21.1 \%})$ which was statistically significant (p 0.0045). Within the CRT group, there was no significant difference in the outcomes within the MWH $>11 \mathrm{~g} / \mathrm{dL}$ and $\mathrm{MWH}$ :10-10.9g/dL sub-groups $(\mathrm{CR}$ rates of $80 \%$ vs. $61.9 \%, \mathrm{p}=0.4285)$. Conclusions: The importance of maintaining a minimum hemoglobin level of 11g/dL during RT is much greater for patients treated with RT alone, than for patients treated with concurrent chemoradiotherapy. Enhanced haemoglobin levels during RT may to an extent negate the ill-effects that may otherwise arise due to non-use of concurrent chemotherapy.
\end{abstract}

Keywords: Hemoglobin during radiotherapy, locally advanced cervical cancer, concurrent chemoradiotherapy

Asian Pac J Cancer Prev, 16 (10), 4285-4289

\section{Introduction}

Radiotherapy has been the mainstay of treatment for locally advanced carcinoma of the cervix (LACC), and the addition of concurrent chemotherapy has been documented to offer better responses. However, LACC is a disease mostly prevalent in developing and underdeveloped economies, where financial and infrastructural constraints may limit the routine use of concurrent chemotherapy with RT. Furthermore, in addition to issues of non-affordability, some patients cannot be treated with concurrent chemotherapy due to inadequate renal function (a common occurrence in stage IIIB LACC due to hydronephrosis), frail general condition, or co-morbidities. Further, few region-specific studies have failed to demonstrate a clear benefit with the addition of concurrent chemotherapy (Negi et al., 2010; Srivastava et al., 2013). Thus, a sizable proportion of LACC patients will continue to be treated with RT alone (without concurrent chemotherapy).

Owing to circumstances prevailing in the institution during the years 2009-2010, patients would be treated with either of RT alone (by altered fractionation in the form of accelerated hyperfractionated radiotherapy) or 


\begin{tabular}{ll}
\hline Inclusion criteria & Exclusion Criterea \\
\hline Age $>18 \&<70$ years & Break during external beam radiotherapy for more than three consecutive days \\
Biospy confirmed squamous cell histology & $\begin{array}{l}\text { Response assessment done beyond } 20 \text { days from completion of external beam } \\
\text { radiotherapy } \\
\text { Ovean weekly hemoglobin not less than } 10 \mathrm{~g} / \mathrm{dL} \\
\text { Of brachytherapy) exceeding } 50 \text { days. } \\
\text { Use of neoadjuvant chemotherapy }\end{array}$ \\
& $\begin{array}{l}\text { Brachytherapy performed during course of external beam RT } \\
\text { Adenocarcinoma and adenosquamous histology } \\
\text { Non availabilty of mean weekly hemoglobin levels } \\
\text { Incomplete records }\end{array}$ \\
\hline
\end{tabular}

with concurrent cisplatin chemoradiotherapy (if the threefold increase in treatment cost would be affordable by patients with no contraindication to cisplatin). Thus, we recognized a unique retrospective opportunity wherein comparisons could be made between patients with the same disease characteristics being treated with either of RT alone (by accelerated hyperfractionated radiotherapy) versus concurrent chemoradiotherapy (CRT).

Haemoglobin levels during RT are considered to be a very important prognostic factor, supposedly second only to stage in importance (Grogan et al., 1999). This is to the best of our knowledge the first ever study which compares the response rates to external beam radiotherapy phase with radiotherapy alone or with concurrent chemotherapy, with regards to the haemoglobin levels. It must be pointed out here that all patients did undergo subsequent intracavitary brachytherapy, and that the assessments of complete response (CR) rates in this study has been restricted only to the external beam radiotherapy phase, since the beneficial effects of subsequent brachytherapy would be common to either group. Further, the assessments made after completion of brachytherapy would have been blind to the early response patterns.

\section{Materials and Methods}

The treatment with external beam RT phase was delivered either with concurrent chemoradiotherapy (weekly cisplatin at $40 \mathrm{mg} / \mathrm{m}^{2}$ along with $45 \mathrm{~Gy}$ delivered over 5 weeks at $1.8 \mathrm{~Gy}$ per fraction) or with altered fractionated RT alone (50Gy over 4 weeks delivered in a accelerated hyperfractionated regimen with 20Gy in 10 fractions over the first two weeks followed by $30 \mathrm{~Gy}$ delivered in $1.5 \mathrm{~Gy}$ twice-daily fractions over the third and fourth treatment weeks).

As per institutional protocol, aggressive measures were in place to ensure a hemoglobin level of atleast $10 \mathrm{~g} / \mathrm{dL}$. This was ensured not only by the use of frequent packed red cells transfusions, but also by the use of oral haematinics in all patients. Weekly hemoglobin was measured each saturday during the course of external beam RT. The arithmetic mean of the weekly hemoglobin (MWH) was calculated for each patient.

Assessment of response to the external beam treatment phase was done prior to the time of subsequent treatment with brachytherapy (within a fortnight from the completion of the external beam RT phase, while ensuring that the overall treatment time did not exceed 7 weeks).
Also, patients treated with early brachytherapy integrated during the course of external beam RT were excluded from this analysis.

Assessments included clinical examinations by two clinicians, and pelvic nodal assessments done via the use of contrast enhanced CT scans. Complete response was to be declared if both clinical and radiological examinations yielded complete regression.

A total of 60 patient records were found fulfilling the eligibility criteria (Table 1). Patients were grouped into either the "CRT group" (those who received concurrent chemoradiotherapy) or the "AHRT group" (those who received accelerated hyperfractionated radiotherapy). In both the AHRT and the CRT groups, patients were subdivided as per the MWH levels ( $\mathrm{MWH}>11 \mathrm{~g} / \mathrm{dL}$ and MWH:10-10.9g/dL sub-groups). Significance cut-off was set at $\mathrm{p}<0.05$, and proportions were compared using the two-tailed Fisher exact test.

\section{Results}

There was no significant difference with regards to the stage-wise distribution or baseline characteristics of patients in the two groups (Table 2). In the AHRT group, $41.4 \%(n=12)$ and $58.6 \%(n=17)$ of the patients belonged to stage IIB and IIIB respectively. In the CCRT group, $35.5 \%(n=11)$ and $64.5 \%(n=20)$ of the patients belonged to stage IIB and IIIB respectively. Similarly the distribution with regards to the MWH of the patients in the two groups was comparable. $65.5 \%(n=19)$ and $67.7 \%(n=21)$ of patients in the AHRT and CCRT groups respectively had $\mathrm{MWH}$ in the range of $10-10.9 \mathrm{~g} / \mathrm{dL}$. While $34.5 \%(n=10)$ and $32.3 \%(n=10)$ of patients in the AHRT

Table 2. Characteristics of Patients in the Two Arms of the Study

\begin{tabular}{lcc}
\hline & AHRT group $(\mathrm{n}=29)$ & CRT group $(\mathrm{n}=31)$ \\
\hline Age & 58 years & 56 years \\
Median & 33-70 years & 29-68 years \\
Range & & \\
Grade & 4 & 6 \\
$\quad$ Well differentiated & 19 & 17 \\
Moderately differentiated & 6 & \\
Poorly differentiated & & 11 \\
Stage distribution & 12 & 20 \\
II & 17 & \\
III &
\end{tabular}

Legend: AHRT: Accelerated hyperfractionated radiotherapy, CRT: concurrent chemoradiotherapy 


\begin{tabular}{llcccc}
\hline & \multicolumn{2}{c}{ AHRT GROUP } & & \multicolumn{2}{c}{ CRT GROUP } \\
\cline { 2 - 5 } & \multicolumn{1}{c}{ CR } & No CR & & CR & No CR \\
\hline MWH 10-10.9g/dL $(\mathrm{n}=19)$ & $4(21.1 \%)$ & $15(78.9 \%)$ & MWH 10-10.9g/dL $(\mathrm{n}=21)$ & $13(61.9 \%)$ & $8(38.1 \%)$ \\
MWH $>11 \mathrm{~g} / \mathrm{dL}(\mathrm{n}=10)$ & $8(80 \%)$ & $2(20 \%)$ & MWH $>11 \mathrm{~g} / \mathrm{dL}(\mathrm{n}=10)$ & $8(80 \%)$ & $2(20 \%)$ \\
$\mathrm{p}$ value 0.0045 & & & $\mathrm{p}$ value 0.4285 & & \\
\hline
\end{tabular}

and CRT groups respectively had $\mathrm{MWH}>11 \mathrm{~g} / \mathrm{dL}$.

The CR rate to the external beam treatment phase for the overall study population was $55 \%$ ( $n=33$ of 60 patients). CR was observed in $41.4 \%(n=12)$ of patients in the AHRT group and in $67.7 \%(n=21)$ of patients in the CRT group $(\mathrm{p}=0.067)$.

As the main intention of this retrospective analysis, the patients in each of the AHRT and the CCRT groups were stratified as per their MWH into two sub-groups- one sub-group with MWH in the range of $10-10.9 \mathrm{~g} / \mathrm{dL}$, or into another sub-group with $\mathrm{MWH}>11 \mathrm{~g} / \mathrm{dL}$. The reason for choosing $11 \mathrm{~g} / \mathrm{dL}$ as the cut-off between the two sub-groups was because that the overall arithmetic mean of the MWH in the study population was $10.98 \mathrm{~g} / \mathrm{dL}$.

When data with regards to the MWH in the AHRT group was analysed in a $2 \times 2$ Table by the Fisher Exact test, it was seen that there was a statistically very significant difference in the CR rate with regards to the MWH. The difference in the $\mathrm{CR}$ rate between the two sub-groups (MWH:10-10.9g/dL versus $\mathrm{MWH}>11 \mathrm{~g} / \mathrm{dL}$ ) within the AHRT group was $21.1 \%$ versus $80 \%(\mathrm{p}=0.0045)$.

When a similar analysis was done for the twosubgroups within the CCRT group (Table 3), the difference in the CR rate was statistically insignificant, though there was a tendency towards better CR rate with increased MWH. The difference in the CR rate between the two sub-groups (MWH:10-10.9g/dL versus $\mathrm{MWH}>11 \mathrm{~g} / \mathrm{dL}$ ) within the CRT group was $61.9 \%$ versus $80 \%$ ( $\mathrm{p}=0.4285$ ).

\section{Discussion}

Carcinoma of the uterine cervix is a major public health problem, more so in the developing Asian countries. It is estimated that of the approximately 274,000 annual deaths due to cervical carcinoma, more than $80 \%$ of these occur in the developing and under-developed countries of the world (Saha et al., 2010). In India, cervical cancer is the leading cause of cancer mortality among women, and India bears about $20 \%$ of the global burdern of cervical carcinoma (Shanta, 2003; Saha et al., 2010). South Asian countries such as India, Sri Lanka and Nepal together contribute to about one-third of the entire global burden of cervical carcinoma (Ferlay et al., 2010; Sathian et al., 2013). It is well documented that a majority of these patients (as high as $75-80 \%$ of the patients) present in advanced stages (National Cancer Registry Programme, 2006; Saha et al., 2010).

Though cervical carcinoma if detected in the early stages is amenable to various curative surgical procedures, the probability of cure decreases with increasing stage. For patients with LACC, radiation based treatments happen to be the main modality of therapy (Das et al., 2011). [Diptimay Das.pdf]. Western studies have supported the use of concurrent cisplatin based chemotherapy to enhance radiotherapeutic outcomes (Green et al., 2005; Wang et al., 2010). [PMID 1603487320157716$]$ However, these results have not always been replicated in Asian patients. Reasons include non-tolerability of chemotherapy, and the frequent presence of compromised renal function which precludes the safe use of platinum based chemotherapy. Few Asian studies have failed to reveal a clear survival benefit from the use of concurrent cisplatin based chemoradiotherapy (Chiara et al., 1994; Thomas et al., 1998; Pearcey et al., 2002). [PMID (PMID 8048390, PMID 9600821 PMID 11844818)]

There exist a large number of reasons which hinder the delivery of concurrent chemotherapy with radiotherapy among Asian patients, which include factors which could be patient related (advanced age, frail general condition, and compromised renal functions which is common in stage IIIB cervical carcinoma) and well as infrastructure related (the lack of medical oncology facilities, non-affordability of drugs and the such). Many clinicians routinely attempt to minimize the ill-effects of not delivering concurrent chemotherapy by radiobiological measures such as accelerated radiotherapy, and hyperfractionated radiotherapy. And given the improtance of adequate hemoglobin levels, most departments have protocols in place so as to maintain a minimum $\mathrm{Hb}$ level of atleast 10g/dL (Vigario et al., 1973; Resbeut et al., 2003; Marchal et al., 2005).

Routine radiotherapy for cervical carcinoma is done with the use of energies in the range of $4 \mathrm{MV}$ to $15 \mathrm{MV}$ $\mathrm{X}$-rays using linear accelerators, or by the use of $1.25 \mathrm{MeV}$ cobalt- 60 gamma rays. The ionizing radiation bearing the mentioned energies are regarded to be of 'low linear energy transfer', and hence having a high 'oxygen enhancement ratio'- meaning that the effect of the particular ionizing radiation will be much higher under adequate oxygenation rather than in the lack of or indequate oxygenation (Hall \& Giaccia, 2006; Wenzl \& Wilkens, 2011).

Tumor hypoxia is a major contributor of nonresponsiveness to radiotherapy. Also, tumor hypoxia can lead to increased propensity for rapid progession and distant metastases (Vaupel et al., 2001). Vaupel et al utilized intratumoral oxygen tension measurements in 59 patients of cervical carcinoma to reveal evidence that intratumoral hypoxia does indeed result from anaemia. They described optimum hemoglobin levels as those between $12-14 \mathrm{~g} / \mathrm{dL}$. It was observed that a blood hemoglobin concentration of $<12 \mathrm{~g} / \mathrm{dL}$ led to compromized tumor oxygenation, while levels $>14 \mathrm{~g} /$ $\mathrm{dL}$ led to a worsening of tumor oxygenation, which they explained as probably due to a drop in perfusion due to viscous resistance to flow. Thus, while low hemoglobin is considered harmful, the risks associated with very high 
levels of hemoglobins should not be overlooked (Vaupel et al., 2002; Vaupel et al., 2006).

Tumor hypoxia can lead to increased angiogenesis due to upregulation of factors such as Hypoxia Inducible Factor -1alpha (HIF-1alpha) (Dellas et al., 2008). This could be illustrated by the observation that poorly oxygenated tumors have higher microvessel density (Dunst et al., 2003). The problem of anaemia among cervix cancer patients is quite ubiquitous. Dunst et al observed pre-treatment hemoglobin as well as midtherapy hemoglobin levels both have an impact, with the latter being of greater significance upon local control and survival (Dunst et al., 2003).

Despite the evidence for better outcomes with improved hemoglobin levels, an exact consensus has not been reached as to the exact minimum level that must be maintained during radiation based treatment for LACC. As per the results of a large Canadian retrospective analysis, the negative effects of anemia during radiotherapy could be remedied by using transfusions to correct hemoglobin levels to $>12 \mathrm{~g} / \mathrm{dL}$ (Grogan et al., 1999). We utilized a cutoff of $11 \mathrm{~g} / \mathrm{dL}$ to stratify patients based on the fact that the median MWH for the entire population was $10.98 \mathrm{~g} / \mathrm{dL}$. This value is also supported by a study from Australia by Kapp et al which suggested a minimum hemoglobin level of atleast 11g/dL (Kapp et al., 2002).

There was an overall higher rate of complete response among patients treated with CRT than with patients treated with AHRT (67.7\% vs 41.4\%, $\mathrm{p}=0.067)$. But as the main intention of the study it was examined if the MWH levels had any relation with the response rates among patients in either arm. In our retrospective sample, there was an overall better CR rate for patients with a $\mathrm{MWH}$ greater than $11 \mathrm{~g} \%$. The CR rate was $80 \%$ versus $42.5 \%$ $(\mathrm{p}=0.0069)$ for patients with $\mathrm{MWH}>11 \mathrm{~g} / \mathrm{dL}$ versus for patients with MWH:10-10.9g/dL, respectively. When analysis for CR rates with regards to $\mathrm{MWH}$ was done for each of the CRT and the AHRT groups, it was observed that an enhanced MWH of atleast $11 \mathrm{~g} / \mathrm{dL}$ was of much greater importance for patients receiving RT alone (AHRT) than in comparison to patients receiving CRT.

The observations imply that the use of concurrent chemotherapy may in a small way mitigate the effects of mild anaemia. However, it could more strongly be stated that for patients treated with a radiotherapy regimen containing no concurrent chemotherapy, a deficit in hemoglobin levels could translate to very poor outcomes.

This study is unique in that response rates to external beam treatments only is assessed. The study was confined to observe the CR rates to external beam RT phase only, rather than to assess response after completion of treatment with brachytherapy, since the benefit with brachytherapy could potentially mask the differences in early response rates between the two groups.

In conclusion, even though concurrent chemoradiotherapy is the standard of care for patients with locally advanced cervical carcinoma, for various reasons certain patients will have to be treated with RT alone. Though there is an overall benefit with improved haemoglobin levels in all patients of LACC being treated with radiation based treatment (radiation alone, or in combination with chemotherapy), the relative benefit of an improved hemoglobin level seems to be much higher for patients treated with RT alone than for with patients treated with concurrent chemoradiotherapy. It is possible that the radiosensitizing effect of improved hemoglobin levels may to an extent compensate for the non-use of concurrent chemotherapy..

\section{References}

Chiara S, Bruzzone M, Merlini L, et al (1994). Randomized study comparing chemotherapy plus radiotherapy versus radiotherapy alone in FIGO stage IIB-III cervical carcinoma. GONO (North-West Oncologic Cooperative Group). Am J Clin Oncol, 17, 294-7.

Das D, Chaudhuri S, Deb AR, et al (2011). Treatment of cervical carcinoma with high-dose rate intracavitary brachytherapy: two years follow-up study. Asian Pac J Cancer Prev, 12, 807-10.

Dellas K, Bache M, Pigorsch SU, et al (2008). Prognostic impact of HIF-1alpha expression in patients with definitive radiotherapy for cervical cancer. Strahlenther Onkol, 184, 169-74.

Dunst J, Kuhnt T, Strauss HG, et al (2003). Anemia in cervical cancers: impact on survival, patterns of relapse, and association with hypoxia and angiogenesis. Int $J$ Radiat Oncol Biol Phys, 56, 778-87.

Ferlay J, Shin HR, Bray F, et al (2010). Cancer incidence and mortality worldwide. GLOBOCAN 2008: IARC cancer Base No.10 [Internet]. Lyon, France: International agency for research on cancer. Available from: http://globocon.iarc. fr. Accessed on Jan 10, 2012.

Green J, Kirwan J, Tierney J, et al (2005). Concomitant chemotherapy and radiation therapy for cancer of the uterine cervix. Cochrane Database Syst Rev, 3, 2225.

Grogan M, Thomas GM, Melamed I, et al (1999). The importance of hemoglobin levels during radiotherapy for carcinoma of the cervix. Cancer, 86, 1528-36.

Hall EJ, Giaccia AJ. Editors: Radiobiology for the radiologist, Lippincott Williams \& Wilkins, 6th Ed., 2006.

Kapp KS, Poschauko J, Geyer E, et al (2002). Evaluation of the effect of routine packed red blood cell transfusion in anemic cervix cancer patients treated with radical radiotherapy. Int $J$ Radiat Oncol Biol Phys, 54, 58-66.

Marchal C, Rangeard L, Brunaud C (2005). Anemia impact on treatments of cervical carcinomas. Cancer Radiother, 9, 87-95.

National Cancer Registry Programme (2006). An overview. Indian Council Med Res, New Delhi.

Negi RR, Gupta M, Kumar M, et al (2010). Concurrent chemoradiation in locally advanced carcinoma cervix patients. J Cancer Res Ther, 6, 159-66.

Pearcey R, Brundage M, Drouin P, et al (2002). Phase III trial comparing radical radiotherapy with and without cisplatin chemotherapy in patients with advanced squamous cell cancer of the cervix. J Clin Oncol, 20, 966-72.

Resbeut M, Fondrinier E, Fervers B, et al (2003) .Standards, Options and Recommendations for the management of invasive cervical cancer patients (non metastastic). Bull Cancer, 90, 333-46.

Saha A, Chaudhury AN, Bhowmik P, Chatterjee R (2010). Awareness of cervical cancer among female students of premier colleges in Kolkata, India. Asian Pac J Cancer Prev, 11, 1085-90.

Sathian B, Fazil A, Sreedharan J, et al (2013). Statistical modelling and forecasting of cervix cancer cases in radiation 

oncology treatment: a hospital based study from Western Nepal. Asian Pac J Cancer Prev, 14, 2097-100.

Shanta V (2003). Perspectives in cervical cancer prevention in India, . The international network for cancer treatment and research.

Srivastava K, Paul S, Chufal KS, et al (2013). Concurrent chemoradiation versus radiotherapy alone in cervical carcinoma: A randomized phase III trial. Asia Pac J Clin Oncol, 9, 349-56.

Thomas G, Dembo A, Ackerman I, et al (1998). A randomized trial of standard versus partially hyperfractionated radiation with or without concurrent 5 -fluorouracil in locally advanced cervical cancer. Gynecol Oncol, 69, 137-45.

Vaupel P, Kelleher DK, Höckel M (2001). Oxygen status of malignant tumors: pathogenesis of hypoxia and significance for tumor therapy. Semin Oncol, 28, 29-35.

Vaupel P, Thews O, Mayer A, Höckel S, Höckel M (2002). Oxygenation status of gynecologic tumors: what is the optimal hemoglobin level? Strahlenther Onkol, 178, 727-31.

Vaupel P, Mayer A, Höckel M (2006). Impact of hemoglobin levels on tumor oxygenation: the higher, the better? Strahlenther Onkol, 182, 63-71.

Vigario G, Kurohara SS, George FW (1973). Association of hemoglobin levels before and during radiotherapy with prognosis in uterine cervix cancer. Radiology, 106, 649-52.

Wang N, Guan QL, Wang K, et al (2011). Radiochemotherapy versus radiotherapy in locally advanced cervical cancer: a meta-analysis. Arch Gynecol Obstet, 283, 103-8. 